\title{
(Rose Bengal)/(Eosin Yellow)-Gold-Polypyrrole Hybrids: A Design for Dual Photo-Active Nano-System with Ultra-High Loading Capacity
}

\author{
Abdullah I El-Kholy (D) \\ Doaa Abdel Fadeel (D)' \\ Maha Nasr (D) ${ }^{2}$ \\ Ibrahim El-Sherbiny $\mathbb{D}^{3}$ \\ Maha Fadel' \\ 'Department of Medical Applications of \\ Laser, Pharmaceutical Nano-Technology \\ Unit, National Institute of Laser \\ Enhanced Sciences (NILES), Cairo \\ University, Giza, Egypt; ${ }^{2}$ Department of \\ Pharmaceutics and Industrial Pharmacy, \\ Faculty of Pharmacy, Ain Shams \\ University, Cairo, Egypt; ${ }^{3}$ Nanomaterials \\ Lab, Center for Materials Science, Zewail \\ City of Science and Technology, 6th \\ October City, Giza, Egypt
}

Purpose: Enhancement of the photodynamic/photothermal efficiency of two water-soluble dyes, rose bengal (RB) and eosin yellow (EY), via conjugation to a polymeric nano-system gold-polypyrrole nanoparticle (AuPpy NPs).

Methodology: A multi-step synthesis method and an in situ one-pot synthesis method were used. Loading percentage, particle size, zeta potential, morphology, UV-Vis-NIR spectrophotometry and in vitro photothermal activity were measured. Then, both hybrid nanocomposites were examined for their cytotoxicity and photocytotoxicity on HepG2 cell line as a model for cancer cells.

Results: Dyes loaded in the traditional multi-step method did not exceed $9 \% \mathrm{w} / \mathrm{w}$, while in the one-pot synthesis method they reached $\sim 67 \% \mathrm{w} / \mathrm{w}$ and $\sim 75 \% \mathrm{w} / \mathrm{w}$ for EY-AuPpy NPs and RB-AuPpy NPs, respectively. UV-Vis-NIR spectrophotometry showed that both nanosystems exhibited intense absorption in the NIR region. The mean size of the nanoparticles was $\sim 31.5 \mathrm{~nm}$ (RB-AuPpy NPs) and $\sim 33.6 \mathrm{~nm}$ (EY-AuPpy NPs) with zeta potential values of $-26.5 \mathrm{mV}$ and $-33 \mathrm{mV}$, respectively. TEM imaging revealed the morphology of both hybrids, showing ultra-nano spherical-shaped gold cores in the case of RB-AuPpy NPs, and different shapes of larger gold cores in the case of EY-AuPpy NPs, both embedded in the polymer film. Conjugation to AuPpy was found to significantly reduce the dark cytotoxicity of both $\mathrm{RB}$ and EY, preserving the photocytotoxicity of EY and enhancing the photocytotoxicity of RB.

Conclusion: Gold-polypyrrole nanoparticles represent an effective delivery system to improve the photodynamic and photothermal properties of RB and EY. The in situ one-pot synthesis method provided a means to greatly increase the loading capacity of AuPpy NPs. While both hybrid nanocomposites exhibited greatly diminished dark cytotoxicity, RBAuPpy NPs showed significantly enhanced photocytotoxicity compared to the free dyes. This pattern enables the safe use of both dyes in high concentrations with sustained action, reducing dose frequency and side effects.

Keywords: polypyrrole nanoparticles, polymerization, nano-system, photosensitizer, photocytotoxicity

\section{Introduction}

Rose bengal and eosin yellow are two xanthene dyes commonly used in photodynamic therapy and photodynamic inactivation of micro-organisms. ${ }^{1-5}$ However, the efficient use of the two dyes, as photosensitizers, faces some pharmaceutical challenges, such as poor lipophilicity and non-selectivity which limits their clinical use. $^{6,7}$ One of the basic aspects of pharmaceutical technology is to overcome the
Correspondence: Maha Fadel

Tel +201001649550

$\mathrm{Fax}+2035729499$

Email mahafmali@niles.edu.eg 


\section{Graphical Abstract}

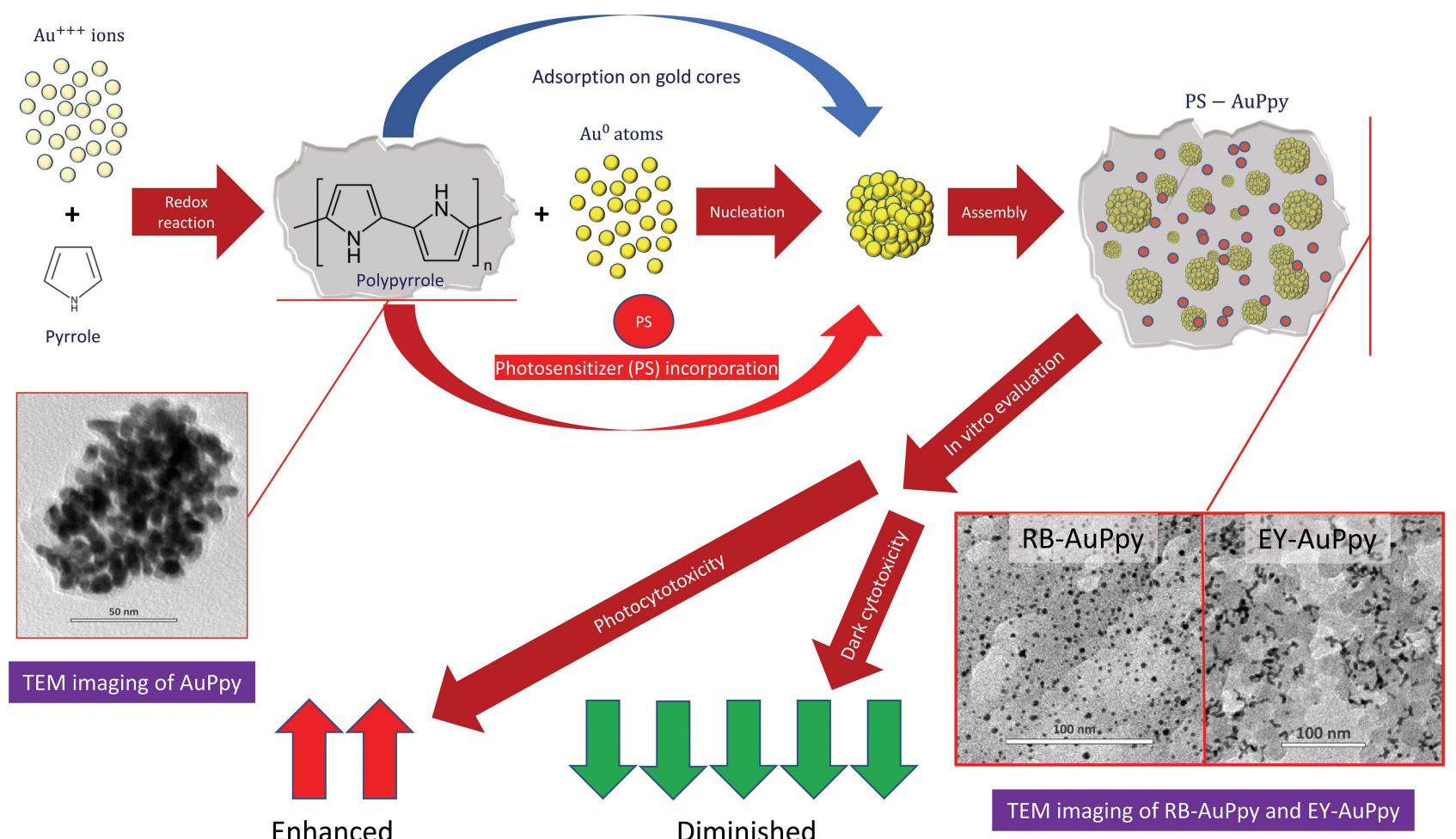

obstructions of optimal drug use such as insolubility, poor bioavailability, high toxicity, poor efficacy, etc. Concurring with the recent advances in pharmaceutical technology, several drug delivery systems were developed and utilized to improve the pharmaceutical properties of numerous drugs, among which are photosensitizers. ${ }^{8-15}$ By the beginning of the 21 st century and in the last two decades, great developments and contributions in the nanotechnology field have given rise to a wide variety of nano-systems based mainly on nanoparticles. ${ }^{16}$ Such nanoparticles are widely variable in their properties and, hence, their purposes and uses. Types of nanoparticles are too many to list, but they can be divided into three basic categories: metal nanoparticles, non-metal nanoparticles and hybrid nanoparticles. Metal nanoparticles are made of pure metals (eg, platinum, silver, gold, titanium) or their compounds (eg, oxides, hydroxides, phosphates). ${ }^{17}$ Axiomatically, nonmetal nanoparticles cover all nanoparticles that are not composed of pure metals or their compounds such as carbon nanotubes and polymeric nanoparticles. On the other side, hybrid nanoparticles, as the name suggests, are those composed of more than one component (metal/ non-metal, metal/metal or non-metal/non-metal). ${ }^{18}$ This diversity enables the development of such materials with pre-engineered and specified properties. Pronounced examples here are magnetic nanoparticles, ${ }^{19,20}$ fluorescent nanoparticles, ${ }^{21}$ stimuli-responsive nanoparticles ${ }^{22}$ and multifunctional hybrid nano-systems, ${ }^{23,24}$ among others. Coinciding with the accelerating pace in this field, polymeric drug delivery systems were recently introduced as platforms for selective and controlled delivery of drugs. ${ }^{25}$ Among polymeric materials, conducting polymers are considered to be promising in formulation of multi-purpose drug delivery systems. ${ }^{26,27}$ Well-known examples of such compounds are polypyrroles, which are polymers with unique conducting properties. ${ }^{28}$ Despite their organic nature, such compounds are characterized by their ability to transfer electrical current to an extent that may exceed those of semi-conductors or even metals. ${ }^{29}$ The hybrid nature of polypyrroles, being organic yet conducting compounds, has delineated them as materials of interest in several fields. ${ }^{30,31}$ Apart from their industrial applications, 
polypyrroles were recently exploited as flexible tools in medicine and pharmacy, especially in photothermal therapy, ${ }^{32}$ biosensors designing, ${ }^{33,34}$ nerve-repairing surgeries $^{35}$ and tailored drug delivery systems. ${ }^{26}$ Photothermal therapy involves the use of a material possessing photothermal properties to induce programmable hyperthermia, leading to specific effect (eg, cell death). ${ }^{36}$

The most commonly used nano-system in this approach is gold nanorods, ${ }^{36,37}$ but owing to its drawbacks which involve synthesis-related coating toxicity and nonbiodegradability, ${ }^{38}$ the use of biocompatible alternative materials with comparable efficiency became a necessity. Polypyrrole is a good example of an alternative material with high biocompatibility and low cytotoxicity, ${ }^{39-41}$ but its challenging pharmaceutical properties (eg, very poor solubility) represent a basic obstacle. ${ }^{42}$ In a previous work, we introduced highly dispersed polypyrrole-coated gold nanoparticles as a novel photothermal agent with high tolerability, compatibility and good pharmaceutical properties. ${ }^{43}$ In this study, rose bengal and eosin yellow, which are well-known photosensitizers, were loaded into the aforementioned nanoparticles, and the resulting loaded nanoparticles were characterized and examined for their cytotoxicity and photocytotoxicity.

\section{Materials and Methods Materials}

Chloroauric acid $\left(\mathrm{HAuCl}_{4}\right)$, rose bengal (RB) and eosin yellow (EY) were purchased from Sigma-Aldrich Co. (St. Louis, USA). Dulbecco's modified Eagle's media (DMEM), fetal bovine serum (FBS), trypsin-EDTA and streptomycin-penicillin $G$ mixture were purchased from Lonza (B-4800 Verviers, Belgium). 3-(4,5-dimethylthiazol-2-yl)-2,5-diphenyl tetrazolium bromide (MTT) was purchased from BIO BASIC CANADA (Ontario, Canada). All other reagents and solvents were of analytical grade.

\section{Loading Rose Bengal and Eosin Yellow Onto AuPpy NPs \\ Post-Synthesis Loading Method}

In this method, RB/EY was added to the already prepared AuPpy NPs as thoroughly described in a previous work by Fadel et al. ${ }^{43}$ Briefly, $1 \mathrm{~mL}$ of $10 \mathrm{mM}$ chloroauric acid was rapidly added to $20 \mathrm{~mL}$ of $25 \mathrm{mM}$ of stirred aqueous solution of pre-distilled pyrrole at room temperature $\left(25^{\circ} \mathrm{C}\right)$, left for $2-3$ min to assure reaction completion, centrifuged at 10,000 rpm for 60 min (Kontron, Italy), and then redispersed in double-distilled water to get the intended concentration. Then, RB/EY was added to the already prepared AuPpy NPs in different concentrations $(100,200$ and $500 \mu \mathrm{M})$, sonicated for 10 minutes, left for 24 hours in a dark cool place and finally centrifuged at $10,000 \mathrm{rpm}$ for one hour to get rid of excess RB and EY.

\section{In-situ One-Pot Synthesis Method}

This method was carried out in one pot in which $1 \mathrm{~mL}$ of $\mathrm{HAuCl}_{4}(10 \mathrm{mM})$ is added rapidly to $20 \mathrm{~mL}$ stirring aqueous pre-distilled pyrrole $(25 \mathrm{mM})$ and RB/EY (100, 200 and $500 \mu \mathrm{M})$ pre-mixed solutions. The reaction was left for 4-5 mins, and the resulting solutions were centrifuged at 10,000 rpm to get rid of excess unreacted RB, EY and pyrrole. Pellets were re-dispersed in double-distilled water to get the intended concentration.

\section{Determination of the Loading Efficiency of RB and EY}

Loading efficiency was determined indirectly from the difference between RB/EY initial concentrations and their concentrations in the supernatant after centrifugation. Concentrations were determined colorimetrically at the maximum visible absorption of both $\mathrm{RB}$ and $\mathrm{EY}$ at 546 $\mathrm{nm}$ and $520 \mathrm{~nm}$, respectively. ${ }^{44,45}$ Loading studies were performed at least in triplicate, and the mean loading values were calculated.

\section{Characterization of the Synthesized Nanoparticles (AuPpy, RB-AuPpy and EY-AuPpy NPs)}

UV-Visible-NIR absorption spectra were measured using a Rayleigh 2601 double-beam spectrophotometer (Beijing, China). Zeta potential and particle size values were measured by a Malvern Zetasizer (Malvern, UK), at least in triplicate. TEM images were captured by a JEOL transmission electron microscope (JEOL, Japan) in order to assess the morphology of the nanoparticles.

\section{Photothermal Study}

The synthesized nanoparticles (AuPpy, RB-AuPpy and EY-AuPpy) in addition to RB and EY solutions and double-distilled water (as a control) were subjected to a photothermal study using $3 \mathrm{~mL}$ of each suspension/ solution of the same concentration (correspondent to 500 $\mu \mathrm{M} R \mathrm{RB}$ or EY photosensitizer in case of RB-AuPpy, EY- 
AuPpy, RB and EY) and the corresponding concentration of AuPpy in case of free AuPpy. Each suspension/solution/ water was irradiated in a Petri dish $(3 \mathrm{~cm}$ in diameter) by white light of a halogen lamp (1 W) for $10 \mathrm{~min}$, and the temperature of the suspension/solution/water was recorded every one min to determine the temperature rise induced by each formulation (nanoparticles suspensions, free dye solutions and water). The photothermal study was repeated at least three times for each, and means were calculated.

\section{Release Study}

Release patterns of RB and EY from RB-AuPpy and EYAuPpy, respectively, were studied over 30 days. In vitro release studies were performed using Visking dialysis tubing with an average flat width of $25 \mathrm{~mm}$ and 12,000 Da MWCO (Medicell Membranes Ltd, UK). One $\mathrm{mL}$ of each formulation of known concentration $(500 \mu \mathrm{M}$ with respect to $\mathrm{RB}$ or $\mathrm{EY}$ ) was placed in the dialysis tubing, sealed, then submerged into $100 \mathrm{~mL}$ of phosphate buffer solution (PBS pH 7.4) in an amber glass container and maintained at $37^{\circ} \mathrm{C} .{ }^{46}$ Concentrations of each dye were detected colorimetrically every $60 \mathrm{~min}$ for $3 \mathrm{~h}$, after 24,48 and $72 \mathrm{~h}$, then after 7, 14, 21 and 30 days. Measured concentrations were plotted as a function of time. Release study was conducted three times, and means were taken.

\section{In vitro Evaluation of Cytotoxicity and Photocytotoxicity \\ Cell Culture}

Human hepatocellular carcinoma (HepG2) cell line (Nawah Scientific Co., Egypt) was used as a model for cancer cells. Cells were cultured on DMEM medium containing $10 \% \mathrm{FBS}$ and $1 \%$ penicillin/streptomycin antibiotic mixture and maintained at $37^{\circ} \mathrm{C}$ under $5 \% \mathrm{CO}_{2}$ humidified atmosphere. ${ }^{47,48}$

\section{Cell Viability Test}

MTT assay was applied to quantify the viability of HepG2 cells, ${ }^{49}$ after treating them with test and control samples for the intended time and conditions. MTT assay was performed by replacing the previous medium with $10 \%$ MTT DMEM medium, ${ }^{46}$ and incubating for $4 \mathrm{~h}$ to allow the reduction of MTT by viable cells. The media were then discarded, and cells were washed by PBS twice. Finally, the formed formazan crystals were solubilized using DMSO $(200 \mu \mathrm{L}$ per well) then read at $570 \mathrm{~nm}$ using ELISA microplate reader (Biotek ELx800, USA).

\section{Cytotoxicity and Photocytotoxicity}

Serial concentrations of free RB and EY $(5,10,20,30,40$, 50,100 and $200 \mu \mathrm{M}$ ) and the corresponding concentrations of the conjugated RB/EY (RB-AuPpy and EY-AuPpy) were examined for their dark cytotoxicity firstly, and then the maximum safe concentrations (ie, the highest concentration that does not exert cytotoxic effect) of both free and conjugated RB/EY were applied for photocytotoxicity experiments. Cells were seeded on culture medium (10\% FBS and $1 \%$ penicillin/streptomycin DMEM medium) in 96-well plates ( $\sim 7000$ cells per well) and left to adhere in the incubator for $24 \mathrm{~h}$ at $37^{\circ} \mathrm{C}$ under $5 \% \mathrm{CO}_{2}$ humidified atmosphere. The old media were then discarded, replaced by $2 \%$ FBS DMEM media containing the test and control samples and left in the incubator for $24 \mathrm{~h}$ under the same conditions. For dark cytotoxicity, MTT assay was applied directly after the incubation period, while, for photocytotoxicity, wells were firstly exposed to white light delivered from a halogen lamp $(1 \mathrm{~W})$ for 5 mins. After irradiation, MTT assay was applied. To avoid errors that may arise from the interference (in visible absorption at $570 \mathrm{~nm}$ ) between RB ( $\lambda_{\max }$ : $540-550$ $\mathrm{nm})$, EY ( $\left.\lambda_{\max }: 520 \mathrm{~nm}\right)$, AuPpy $\left(\lambda_{\max }: 560 \mathrm{~nm}\right)$, different nanoparticles formulations and solubilized formazan (DMSO-solubilized reduced MTT crystals, $\lambda_{\max }: 570 \mathrm{~nm}$ ), negative-MTT control wells were also included where all steps were applied except MTT step. Although the residual contents of formulations in cells were too small to affect the MTT results after solubilization, negative control wells were necessary to exclude any errors especially at high concentrations. All experiments were carried out simultaneously, and each experiment was repeated at least three times.

\section{Statistical Analysis}

Statistics were analyzed by one-way analysis of variance test (one-way ANOVA) followed by Tukey-Kramer test using GraphPad Prism version 5.01 software (GraphPad, San Diego, CA). Differences were considered significant when the $p$-value is less than 0.05 . Results were displayed as means \pm SEs or means \pm SDs.

\section{Results and Discussion}

\section{Loading of Rose Bengal and Eosin Yellow into AuPpy and Determination of the Loading Efficiency}

In case of the conventional multi-step method (ie, postsynthesis method), AuPpy NPs were firstly synthesized. The reaction between $\mathrm{HAuCl}_{4}$ and pyrrole was fast with 
rapid change in the solution color from transparent colorless to deep black color. The synthesized AuPpy nanoparticles were extremely well-dispersed with clear appearance. No aggregates or flocculates were observed in any stage of the reaction. In this reaction, while gold salt $\left(\mathrm{HAuCl}_{4}\right)$ induces the oxidative polymerization of pyrrole, gold ions are reduced into elemental gold (gold atoms). During the nucleation of gold atoms, the synthesized polypyrrole acts as stabilizer for the nucleating gold nanoparticles preventing them from further growth or aggregation. The size of the synthesized gold nanoparticles depends on the initial concentration of the monomer (pyrrole). ${ }^{43}$ The loading efficiency after incubation of RB and EY for $24 \mathrm{~h}$ did not exceed $9 \% \mathrm{w} / \mathrm{w}$ at its best. In contrast, the loading percentages of RB and EY reached $74.6 \pm 1.8 \% \mathrm{w} / \mathrm{w}$ and 67.64 $\pm 2.1 \%$ of the total conjugate, respectively, for the $500 \mu \mathrm{M}$ loading solution, and were $37.24 \pm 0.81 \%$ and $54.04 \pm 0.93 \%$ for $\mathrm{RB}$, and $29.48 \pm 0.74 \%$ and $45.46 \pm 0.86 \%$ for $\mathrm{EY}$, at concentrations $100 \mu \mathrm{M}$ and $200 \mu \mathrm{M}$, respectively (Table 1). This vast difference in loading capacity between the post-synthesis method and the in situ one-pot method can be explained in the light of the loading pattern, where loading, in the first case, takes place just onto the surface of the synthesized nanoparticles (on the outer surfaces of the polypyrrole layer(s) which are of very limited area), while, in the second case, proposed and used in the current work, loading takes place during the synthesis process; hence the dye $(\mathrm{RB} / \mathrm{EY})$ is incorporated within the whole nanoparticle (AuPpy) in addition to the surface (both within and onto the surfaces of the polypyrrole layers), which massively increases the loading capacity. This delineates that almost all the photosensitizers' amounts were loaded within AuPpy, suggesting the high efficiency of this method. Knowing that the real molar ratio of the reaction here is 1:1:2 (RB/EY:Au:Pyrrole) where the excess of pyrrole does not react ${ }^{43}$ and the molecular weights of RB/EY, Au and pyrrole are 973.67/691.86 g/mol, $196.96657 \mathrm{~g} / \mathrm{mol}$ and $67.09 \mathrm{~g} / \mathrm{mol}$, respectively, a question may arise about the capacity of the synthesized polypyrrole to enclose all the synthesized gold nanoparticles plus RB/EY. But a simple comparison between the density of $\mathrm{Au}$ and polypyrrole $\left(19.3 \text { g.cm }{ }^{-3} \text { and } 1.5 \text { g.cm }{ }^{-3}\right)^{50,51}$ reveals that polypyrrole volume alone represents about 13 -fold that of gold nanoparticles. Adding that to the volume of RB/EY already loaded in the polypyrrole film clearly explains why RB/ EY-polypyrrole films totally coat/embed gold nanoparticles in both formulations.

\section{Characterization of AuPpy, RB-AuPpy and EY-AuPpy NPs}

\section{UV-Vis-NIR Spectrophotometry}

As shown in Figure 1, it can be observed that the conjugation dramatically enhanced the NIR absorption of both RB-AuPpy NPs and EY-AuPpy NPs in comparison to the absorption of RB, EY or AuPpy alone, with distinctive new definite peaks in this region $(800-1000 \mathrm{~nm})$. Such peaks were not present in the individual spectra of free AuPpy NPs, but appear very faintly in the spectra of free $\mathrm{RB}$ and free EY, and are more pronounced in case of EYAuPpy than in RB-AuPpy NPs. Taking into consideration that the basic absorption peak of RB and EY are resident in the visible region (546 and $515 \mathrm{~nm}$, respectively) and that of AuPpy nanoparticles nearly covers the whole spectrum, enhancement of the RB/EY-related peak is expected since AuPpy absorption constructively interferes with those of RB and EY in the visible region, but, unevenly, the absorption intensity of both RB and EY in the visible region is diminished upon loading. This may be attributed to the hindering effect of polypyrrole as concluded from a separate experiment, where physical mixtures of both dyes with polypyrrole (in the same concentrations used in the loading process) were prepared and measured for their UV-Vis-NIR absorption. The resulting spectra showed that visible absorption of both dyes was magnified with no effect on the NIR region. This is also an indication that most loading in the presented in situ model was within the polymer not onto the surface. Similarly, the enhancement of RB-AuPpy and EY-AuPpy NIR absorption is somehow

Table I Efficiency of Loading in Both the Conventional Method and the One-Pot Synthesis Method (Values are Expressed as Percentages \pm SDs $w / w)$

\begin{tabular}{|l|c|c|c|c|c|c|}
\hline \multirow{2}{*}{ Type and Concentration $(\mu \mathrm{M})$ of the Photosensitizer } & \multicolumn{3}{|c|}{ Rose Bengal } & \multicolumn{3}{c|}{ Eosin Yellow } \\
\cline { 2 - 7 } & 100 & 200 & $\mathbf{5 0 0}$ & 100 & 200 & 500 \\
\hline Conventional method & $7.4 \pm 0.23$ & $8.3 \pm 0.98$ & $8.6 \pm 3.46$ & $4.2 \pm 0.74$ & $6.82 \pm 0.05$ & $8.13 \pm 2.4$ \\
\hline One-pot synthesis method & $37.24 \pm 0.81$ & $54.04 \pm 0.93$ & $74.6 \pm 1.8$ & $29.48 \pm 0.74$ & $45.46 \pm 0.86$ & $67.64 \pm 2.1$ \\
\hline
\end{tabular}




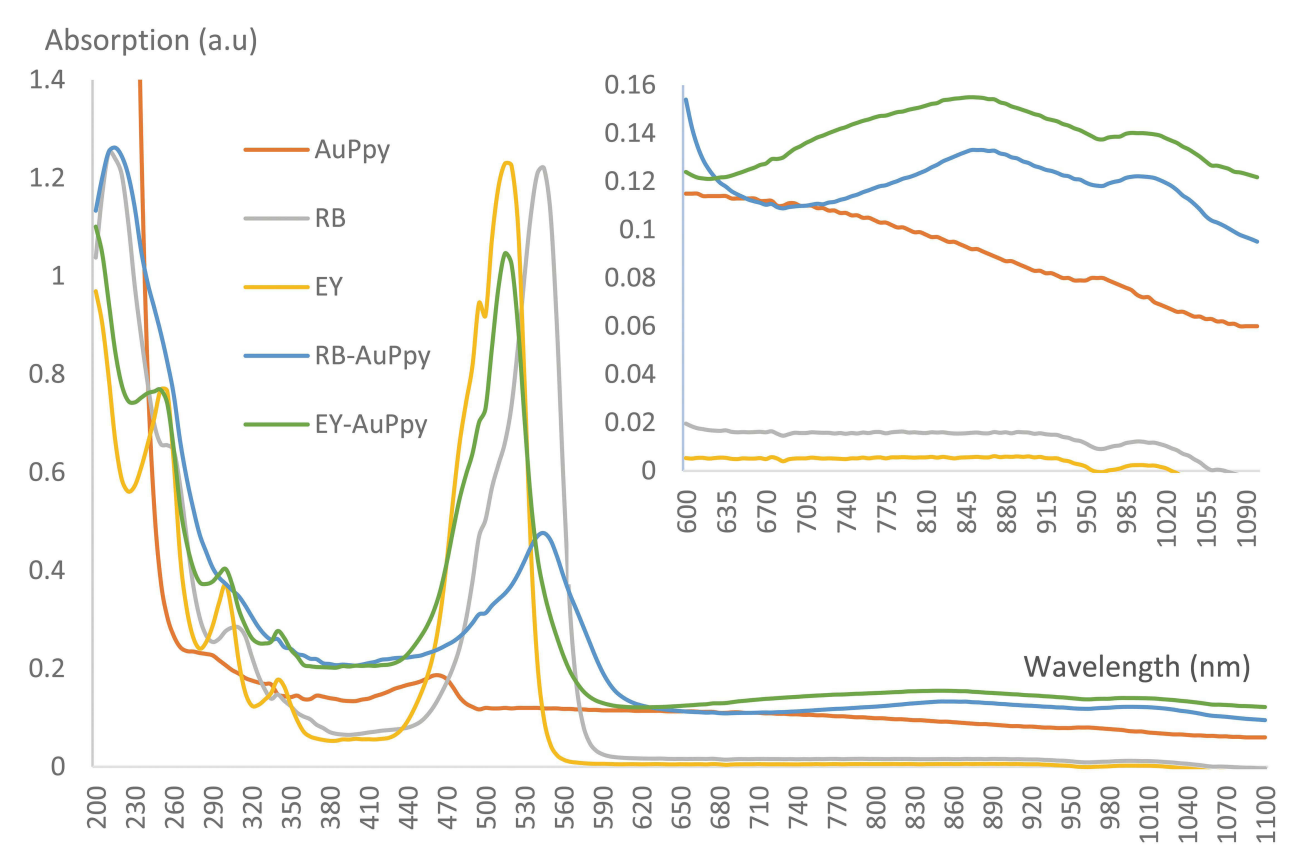

Figure I UV-Vis-NIR absorption spectra of AuPpy, RB-AuPpy and EY-AuPpy NPs (identical diluted concentrations).

unpredictable, since free $\mathrm{RB}$ and $\mathrm{EY}$ have almost no absorption in this region. As reported in similar cases, ${ }^{52}$ gold-polypyrrole conjugation may enhance RB and EY absorption in the NIR region. Another hypothesis involving chemical rather than physical incorporation of some $\mathrm{RB}$ and $\mathrm{EY}$ in the polypyrrole backbone (ie, doping) may be true. This means that conjugation of RB/EY to AuPpy has altered the spectroscopic properties of the gold-polypyrrole conjugate, similar to what was reported by Shrikrushna et al and Rahaman et al. ${ }^{53,54}$ The release pattern of both dyes from conjugates (see below) suggests a hybrid model, where some RB/EY is incorporated in the polymer backbone during synthesis, and the major amount is physically loaded.

\section{Particle Size and Zeta Potential Values}

The zeta potential value of AuPpy was $+46.2 \pm 11.1 \mathrm{mV}$ with average diameter of $56.64 \pm 30.02 \mathrm{~nm}$, while the zeta potential values of RB-AuPpy and EY-AuPpy were $-26.5 \pm 5.41 \mathrm{mV}$ and $-33 \pm 6.36 \mathrm{mV}$ with average diameter of $31.51 \pm 11.93 \mathrm{~nm}$ and $33.63 \pm 25.93 \mathrm{~nm}$, respectively. The high zeta potential values indicate relatively good colloidal stability. ${ }^{46}$ The reversal of the zeta potential values from positive in case of AuPpy to negative in case of RB-AuPpy and EY-AuPpy further confirms the conjugation of the dyes to the nanoparticles, since RB and EY ions bear negative charges, as reported in the conjugation of polypyrrole to allicin (a molecule that mainly results from the enzymatic hydrolysis of alliin). ${ }^{22,55} \mathrm{~A}$ similar pattern was observed by Wen et al upon mixing polypyrrole nanoparticles (zeta potential value: $+37.7 \mathrm{mV}$ ) with silver nanoparticles (zeta potential value: $-35.6 \mathrm{mV}$ ). ${ }^{56}$ This also gives an insight into the mechanism of conjugation between RB/ $\mathrm{EY}$ (or at least a proportion of $\mathrm{RB} / \mathrm{EY}$ ) and polypyrrole that resembles the doping process in polypyrrole. Doping is a process where negatively charged ions such as chloride (small ion) or dodecylbenzenesulphonate (large ion) are incorporated as dopants, mainly to enhance polypyrrole electrical conductivity. ${ }^{57}$ Here, proportions of the loaded RB and EY, being negatively charged large molecules, act as dopants during the synthesis process. This explains the dramatic increase in the NIR absorption of the conjugate, since doping, particularly with large molecules like RB $(973.67 \mathrm{~g} / \mathrm{mol})$ and EY (691.86 g/mol), greatly enhances electrical conductivity, ${ }^{58}$ which, in turn, is reflected in enhancing absorption in the NIR region. ${ }^{59}$

\section{TEM Imaging}

TEM imaging of free AuPpy revealed the previously literature-described morphology of the synthesized hybrid nanoparticles ${ }^{43}$ which resembles spheres-embedded gel masses with a mean diameter of 50-60 nm for each cluster. Individual gold nanoparticles range between $4 \mathrm{~nm}$ and $10 \mathrm{~nm}$ in diameter with spherical or semi-spherical shapes 
(Figure 2A). On the other hand, RB-AuPpy NPs under TEM appear to be quite different showing ultra-nano spherical gold cores $(\sim 2-5 \mathrm{~nm}$ in diameter) relatively more distant from each other, embedded in RB-Ppy films with homogeneous distribution (Figure 2B). Surprisingly, TEM imaging showed that the EY-AuPpy nanoparticles have a completely different morphology with spherical, semi-spherical, short rod-like and crescent-like gold nanoparticles embedded in EY-polypyrrole films (Figure 2C). Gold nanoparticles remain distant from each other as in case of RB-AuPpy but the size of gold cores in case of EYAuPpy is larger than in RB-AuPpy yet still smaller or at least similar to it in AuPpy. This difference in size of the gold nano-cores between AuPpy, RB-AuPpy and EYAuPpy can be explained. The relationship between the size of the synthesized gold nanoparticles and the concentration of the stabilizer is often a parabolic relationship, where size decreases by increasing the concentration of the stabilizer till reaching a minimum, then increase by increasing concentration. ${ }^{60,61}$ Stabilizer here is Ppy, RBPpy or EY-Ppy. So, decrease or increase in size (compared to AuPpy) is predictable depending on the concentration of the synthesis solution and on whether that concentration lies right or left of the concentration corresponding to the vertex of the parabola. If the concentration lies left of that corresponding to the vertex, the size will be reduced by increasing concentration and vice versa. Intuitively, the vertex concentration differs from one compound (stabilizer) to another. This explains the differences in gold core size between preparations.

\section{Photothermal Study}

Among all formulations and from a baseline temperature of $20^{\circ} \mathrm{C}$, the maximum photothermal actions accompanied with the fastest heating rates were exerted by EY-AuPpy, RBAuPpy and free AuPpy where the maximum temperatures reached were $45.7^{\circ} \mathrm{C}, 42^{\circ} \mathrm{C}$ and $41.7^{\circ} \mathrm{C}$ after $10 \mathrm{~min}$, respectively, while the minimum photothermal actions and slowest heating rates (in ascending order) were exerted by water, free $\mathrm{RB}$ and free $\mathrm{EY}$, where the maximum temperatures reached were $27^{\circ} \mathrm{C}, 29.5^{\circ} \mathrm{C}$ and $29.9^{\circ} \mathrm{C}$ after $10 \mathrm{~min}$, respectively. This behavior coincides with the NIR absorption pattern of AuPpy, RB-AuPpy and EY-AuPpy, where maximum absorption is exhibited by EY-AuPpy, while AuPpy and RB-AuPpy exhibit close absorption intensity with the minimum absorption ascribed to AuPpy. Approximating the temperature rise

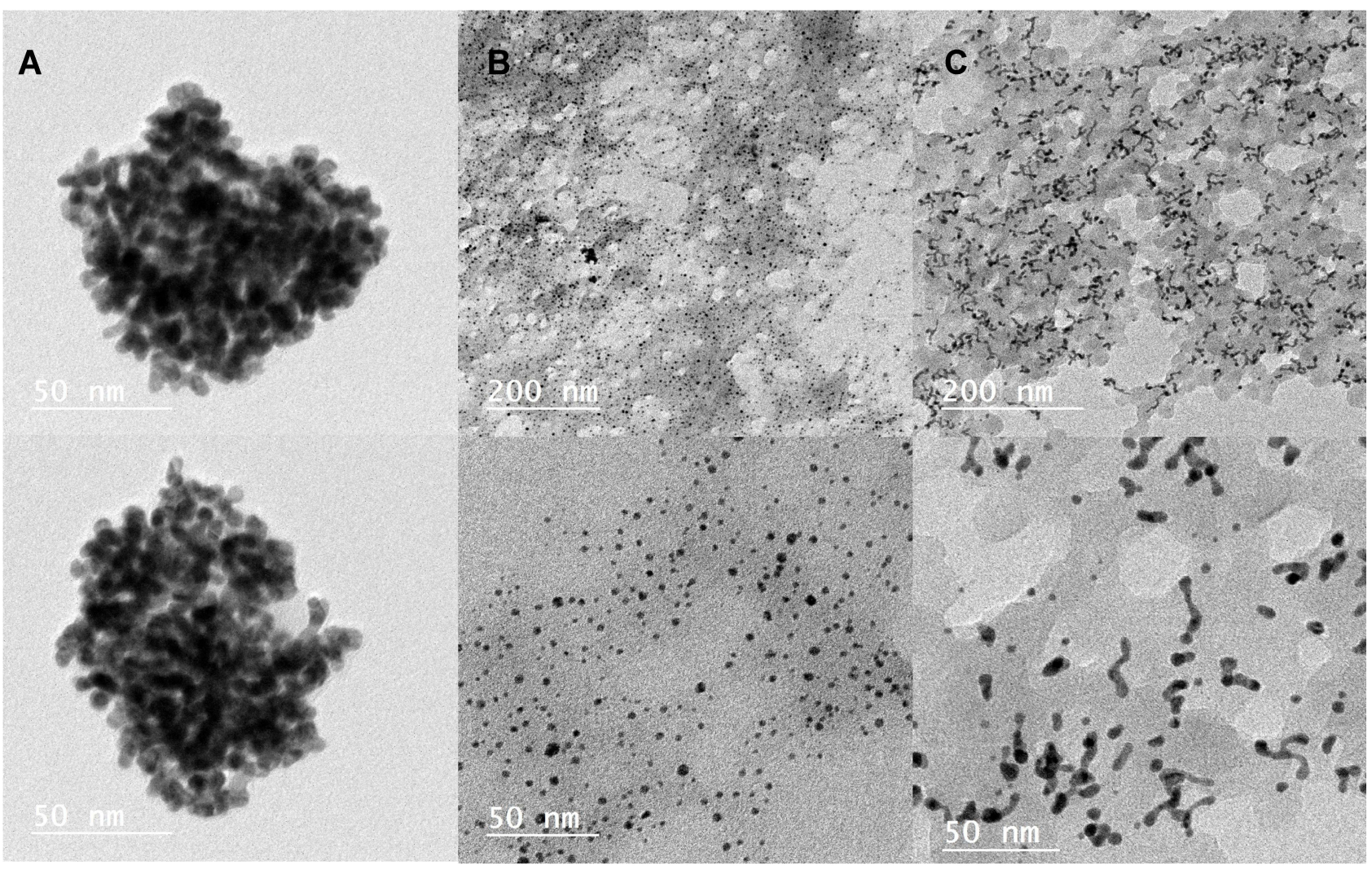

Figure 2 TEM imaging of (A) AuPpy, (B) RB-AuPpy and (C) EY-AuPpy NPs. 
rate equations to the corresponding most probable equations reveals that heating rates of water, free $\mathrm{RB}$ and free $\mathrm{EY}$ follow a logarithmic relationship, while heating rates of AuPpy, RB-AuPpy and EY-AuPpy follow a polynomial relationship. The enhancement of photothermal action in both RB-AuPpy and EY-AuPpy compared to AuPpy enables the use of such composites as multi-purpose drug delivery systems with high loading capacity and photothermal and photodynamic actions. Such enhancement also supports the enhanced absorption in the NIR region. Figure 3 comparatively illustrates the photothermal actions exerted by each formulation. This photothermal action is of particular importance in cancer treatment, in which, when administered simultaneously with chemotherapy or radiotherapy, it results in enhancement of the treatment outcome. ${ }^{62}$

\section{Release Study}

Release of both dyes was slow with initial burst release of $23 \%(\mathrm{RB})$ and $14 \%(\mathrm{EY})$ of the total amount loaded in the first hour followed by gradual slow regular release till reaching maximum concentration of $75 \%$ of the total loaded RB and EY in the accepting solution after 14 and 10 days, respectively (Figure 4). Equations of time-release curves were approximated to the corresponding most probable mathematical equations and found to express a logarithmic relationship at best fitting $\left(\mathrm{R}^{2}\right.$ values are 0.9729 and 0.9568 for RB and EY, respectively). This means that release pattern of both dyes from the polymeric matrix mostly follows a first-order model. Extending the release study to 30 days did not result in significant change in the release of the dyes $(P>0.05)$, and a plateau was reached, which suggests, as mentioned before, the presence of traces of the dyes inside the AuPpy nanoparticles. These traces are probably trapped into the deeper polymer layers (hence making them difficult to be released) or caught by polypyrrole as dopants. However, the latter assumption is more likely since highmolecular-weight dopants are difficult to be released from polypyrrole even under electric potential. ${ }^{63}$ Considering that $\mathrm{RB}$ and EY are relatively large molecules, their incomplete release is justified.

\section{Cytotoxicity and Photocytotoxicity}

As shown in Figure 5, significant differences in cytotoxicity at dark conditions between the photosensitizing dyes $(\mathrm{RB} / \mathrm{EY})$ in the free and loaded form were obvious $(P<0.05)$. The highest safe concentration of free $\mathrm{RB}$ in dark was about $5 \mu \mathrm{M}$, while that of free EY was about $50 \mu \mathrm{M}$. Loading into AuPpy nanoparticles greatly decreased the dark cytotoxicity (ie, enhanced the safety) of both dyes, in which the highest safe concentrations of $\mathrm{RB}$ and EY (conjugated) in dark conditions became about $100 \mu \mathrm{M}$ and $200 \mu \mathrm{M}$, respectively. The enhanced safety of

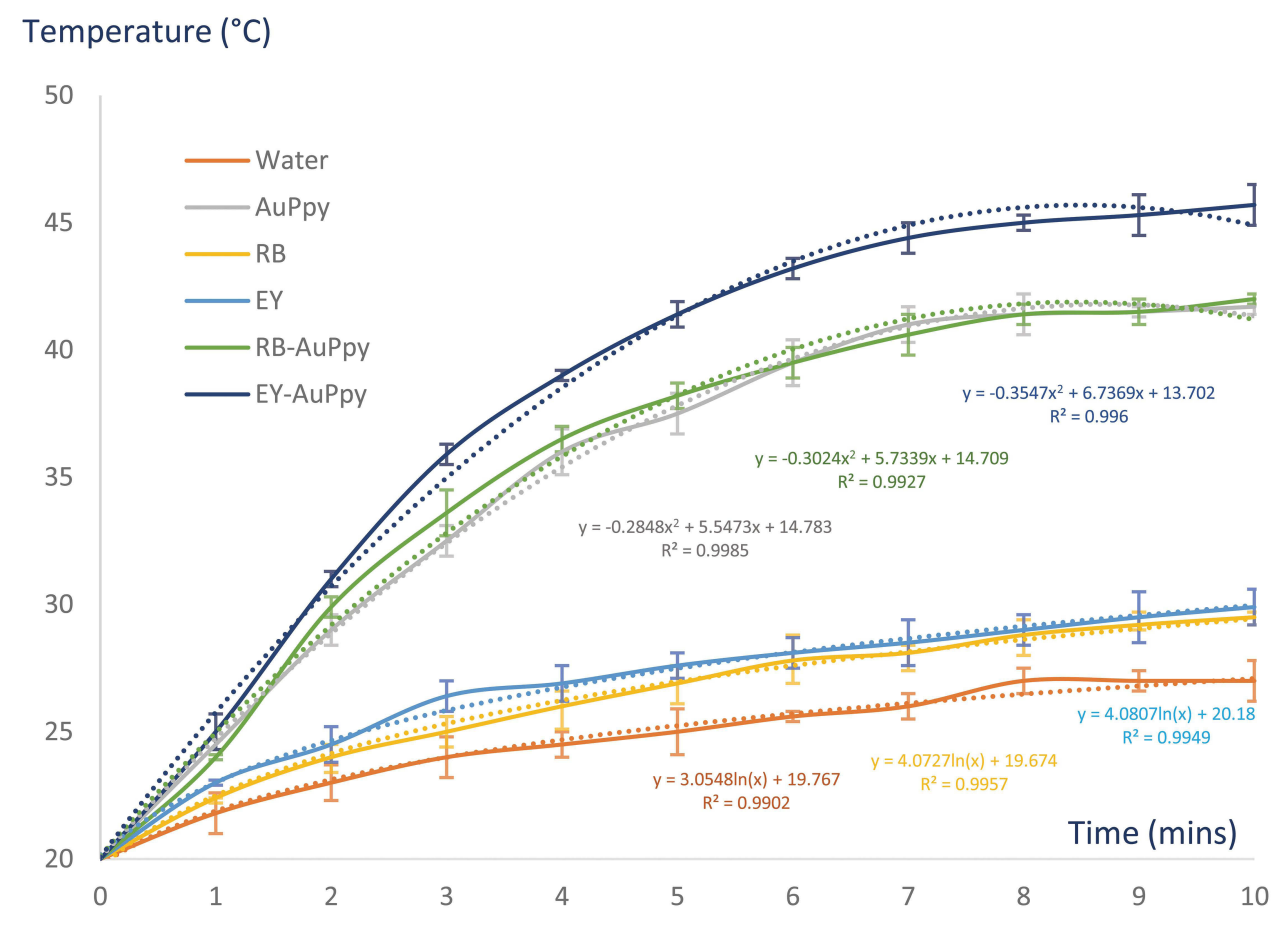

Figure 3 In vitro photothermal activity of water, AuPpy, RB, EY, RB-AuPpy and EY-AuPpy (Means \pm SDs). 


\section{$\%$ Cumulative release}

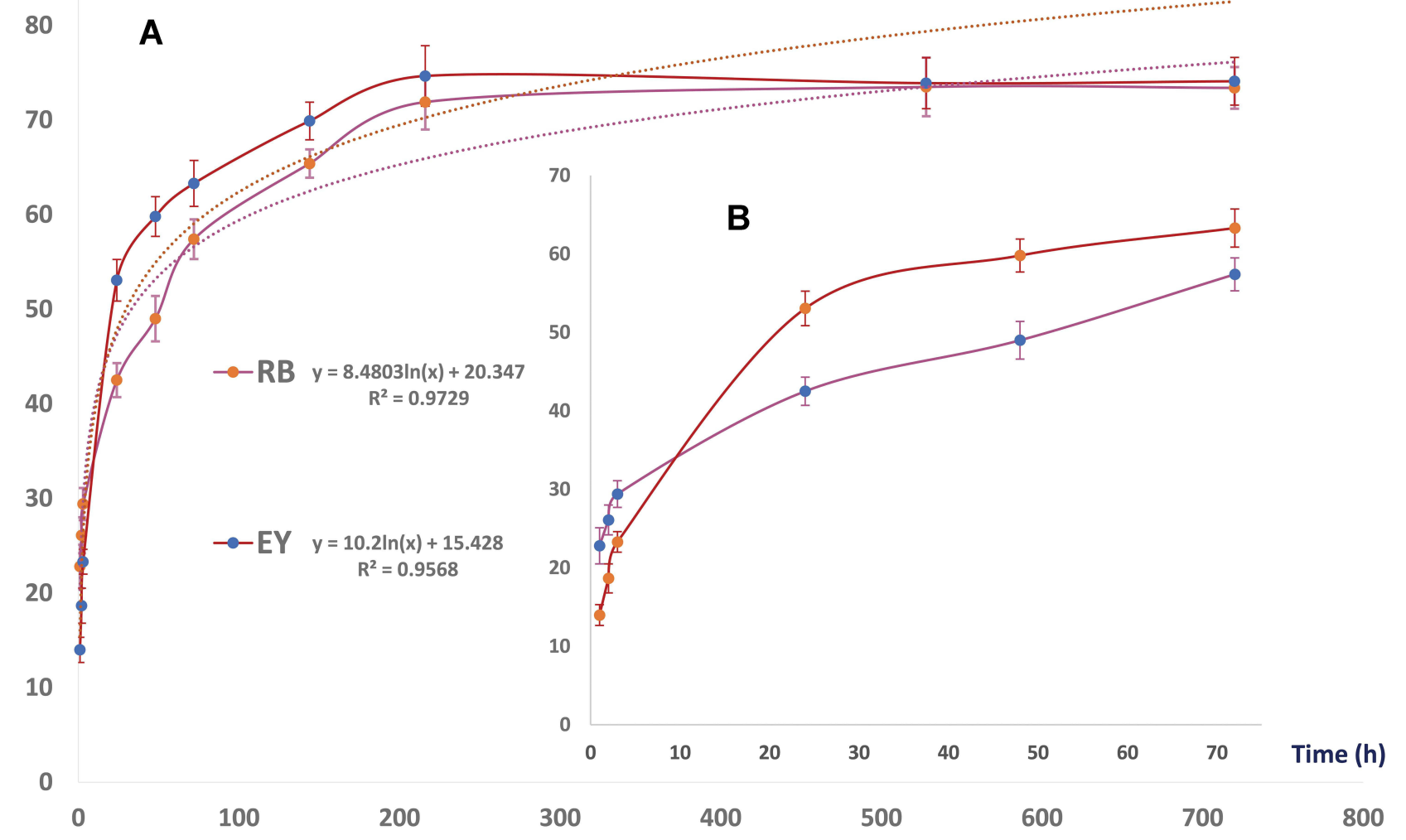

Figure 4 Release pattern of RB and EY from AuPpy NPs (A) over 30 days and (B) over $72 \mathrm{~h}$ (Means \pm SDs).
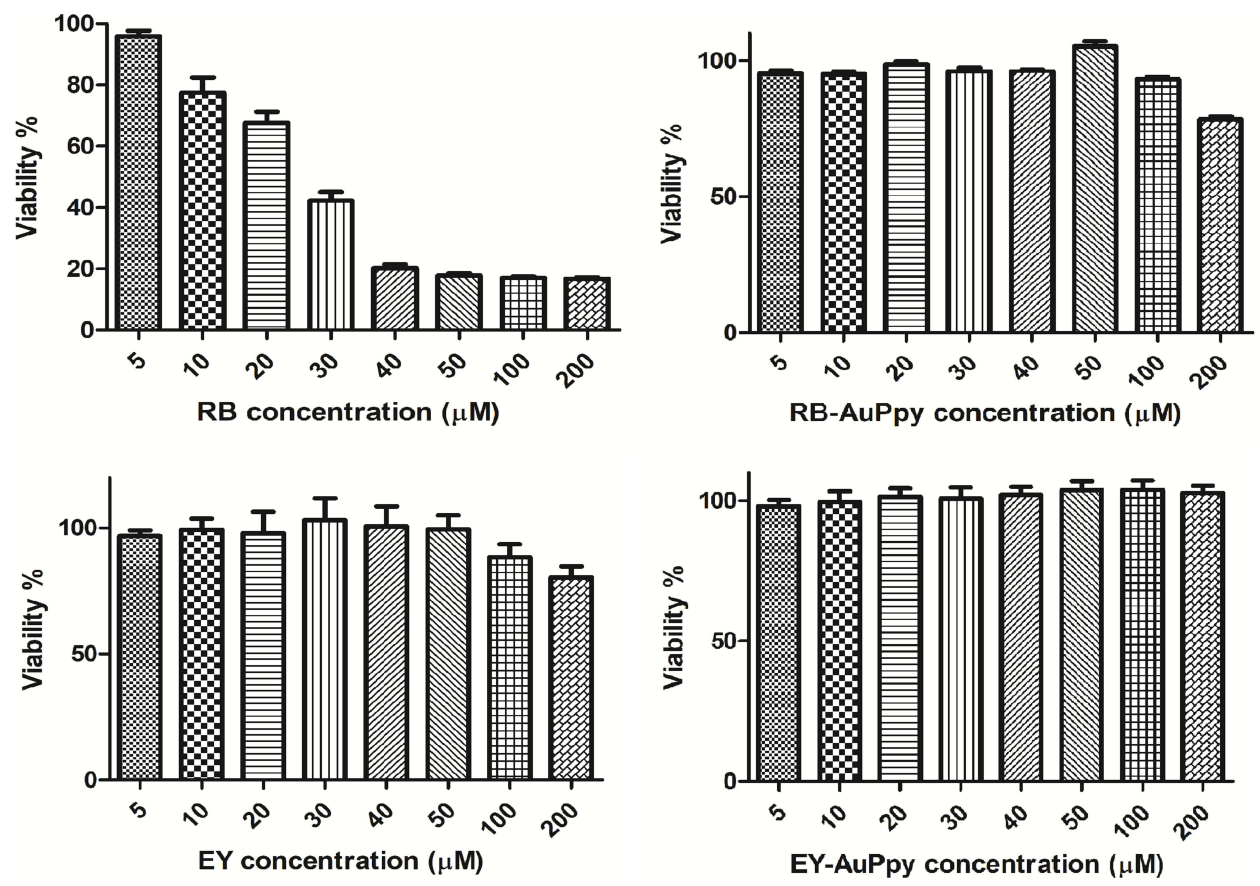

Figure 5 Dark cytotoxicity of RB, EY, RB-AuPpy and EY-AuPpy (Means \pm SEs). 
the dyes by conjugation to AuPpy NPs may be ascribed to the slow release of the dyes, hence decreasing their harmful effects on the cells in dark conditions, ${ }^{64,65}$ which enables controlling and taming their dark cytotoxicity. On the contrary, under irradiation conditions, it was observed that the photocytotoxicity of the conjugated dyes at the highest safe concentrations was the same as that of the free dye (EY-AuPpy) or even higher (RBAuPpy) (Figure 6). Apparently, the cytotoxicity of both conjugates (RB-AuPpy and EY-AuPpy) may be considered of low significance. But a simple comparison between the real available concentration of either dye in case of free and conjugated forms (ie, about $50 \%$ after $24 \mathrm{~h}$ ) clearly reveals the differences in cytotoxicity. Since the release of $\mathrm{RB} / \mathrm{EY}$ from either formulation is somehow slow, this enhancement in photocytotoxicity may be attributed either to the enhanced photothermal action of the whole system or to the enhanced cellular uptake, owing to AuPpy conjugation, of the dyes. Although efficient photothermal action needs a much higher dose of light, the dose applied was enough to induce mild hyperthermia which might have accelerated the action of RB and EY. ${ }^{66-68}$ Another factor that may explain the enhanced photocytotoxicity despite the transient decrease of the free dye concentration (ie, due to slow release) is the efficient cellular uptake of polypyrrole, ${ }^{69}$ which is translated into an overall elevation of the dye concentration after release. Therefore, conjugation to AuPpy achieved two purposes: firstly, minimizing the dark cytotoxicity; and secondly, maximizing the photocytotoxicity of dyes. In other words, the reduced dark cytotoxicity upon conjugation allows the safe use of high concentrations of both photosensitizers with controlled release. The dual nature of the dye-loaded nanoparticles (photodynamic/photothermal) allows the use of the whole

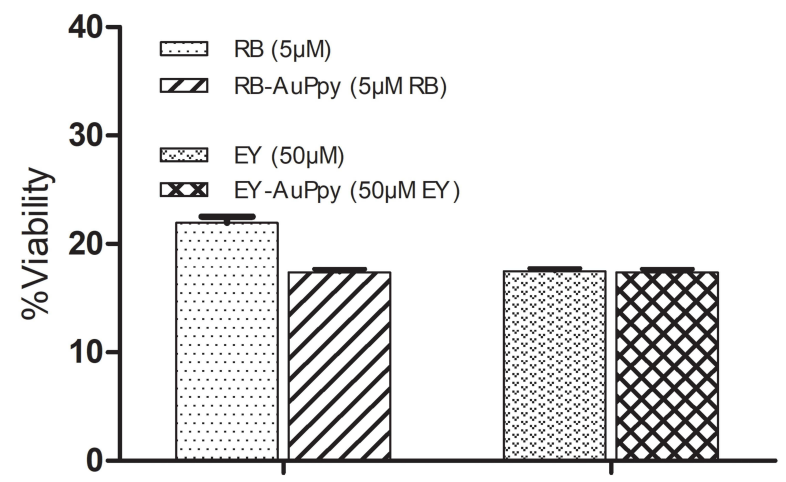

Irradiation for 5 minutes under $1 \mathrm{~W}$ halogen lamp

Figure 6 Photocytoxicity of RB, EY, RB-AuPpy and EY-AuPpy (Means \pm SEs). nano-system as an efficient photodynamic/photothermal tool (the use of the loaded dye as a photodynamic tool and the use of the depleted gold-polymer nanoparticles after dye release as a pure photothermal tool), but this should be applied later since the energy needed to exert photothermal action of polypyrrole is much higher than that needed to activate the photodynamic action of the conjugated/released dyes. Moreover, the slow release of dyes from the AuPpy system makes it possible to repeat the photo-treatment without the need of repeated doses.

\section{Conclusion}

Rose bengal and eosin yellow were successfully loaded within AuPpy with ultra-high loading efficiency in a one-pot simple method. The presence of gold-polypyrrole as a drug delivery system diminished the dark cytotoxicity of both RB and EY, making them controllable and safer for use even at high concentrations, while the apparent photocytotoxicity was enhanced (RB-gold-polypyrrole) or at least was not affected (EYgold-polypyrrole). On the other hand, loading RB and EY within AuPpy significantly enhanced the photothermal activity of the resulting composites compared to free AuPpy. The massive reduction in dark cytotoxicity together with controlled release represents an advantage for clinical use where a single large dose with multiple irradiation sessions could replace multiple dose treatment. Further preclinical and clinical studies are recommended here. In summary, conjugation of both RB and EY to AuPpy enhanced both photothermal action of AuPpy and photodynamic action of RB/EY which, in turn, improves their characteristics as anti-cancer tools. The loading method, used in this study, can be used to easily synthesize other hybrids by changing the loaded drugs.

\section{Abbreviations}

$\mathrm{RB}$, rose bengal; EY, eosin yellow; Ppy, polypyrrole; AuPpy, gold-polypyrrole; NPs, nanoparticles; NIR, near infra-red; UV, ultraviolet, PS; photosensitizer.

\section{Disclosure}

The authors declare no conflicts of interest in this work. This work is supported by a Cairo University project 2016 entitled: "Nano-systems in topical photodynamic therapy and photodynamic diagnosis: preparation, characterization and clinical evaluation". 


\section{References}

1. Panzarini E, Inguscio V, Dini L. Overview of cell death mechanisms induced by rose bengal acetate-photodynamic therapy. Int J Photoenergy. 2011;2011:713726. doi:10.1155/2011/713726

2. Naranjo A, Arboleda A, Martinez JD, et al. Rose bengal photodynamic antimicrobial therapy for patients with progressive infectious keratitis: a Pilot Clinical Study. Am $J$ Ophthalmol. 2019;208:387-396. doi:10.1016/j.ajo.2019.08.027

3. Buck STG, Bettanin F, Orestes E, et al. Photodynamic efficiency of xanthene dyes and their phototoxicity against a carcinoma cell line: a Computational and Experimental Study. $J$ Chem. 2017;2017:7365263. doi:10.1155/2017/7365263

4. Freire F, Borges Pereira da Costa AC, Pereira C, et al. Comparison of the effect of rose bengal- and eosin Y-mediated photodynamic inactivation on planktonic cells and biofilms of Candida albicans. Lasers Med Sci. 2013;29:949-955. doi:10.1007/s10103-013-1435-x

5. Estevão BM, Pellosi DS, de Freitas CF, et al. Interaction of eosin and its ester derivatives with aqueous biomimetic micelles: evaluation of photodynamic potentialities. J Photochem Photobiol a Chem. 2014;287:30-39. doi:10.1016/j.jphotochem.2014.04.015

6. Demartis S, Obinu A, Gavini E, et al. Nanotechnology-based rose bengal: a broad-spectrum biomedical tool. Dyes Pigm. 2021;188:109236. doi:10.1016/j.dyepig.2021.109236

7. Gao Y, Li Z, Wang C, et al. Self-assembled chitosan/rose bengal derivative nanoparticles for targeted sonodynamic therapy: preparation and tumor accumulation. RSC Adv. 2015;5(23):17915-17923. doi:10.1039/C4RA15347B

8. Fadel M, Samy N, Nasr M, et al. Topical colloidal indocyanine green-mediated photodynamic therapy for treatment of basal cell carcinoma. Pharm Dev Technol. 2017;22(4):545-550. doi:10.3109/ 10837450.2016.1146294

9. Ali MFM. Topical delivery and photodynamic evaluation of a multivesicular liposomal rose bengal. Lasers Med Sci. 2011;26 (2):267-275. doi:10.1007/s10103-010-0859-9

10. de Lima Santos H, Fortes Rigos C, Cláudio Tedesco A, et al. Rose bengal located within liposome do not affect the activity of inside-out oriented Na, K-ATPase. Biochim Biophys Acta Biomembr. 2005;1715 (2):96-103. doi:10.1016/j.bbamem.2005.07.014

11. Samy N, Fadel M. Topical liposomal rose bengal for photodynamic white hair removal: randomized, controlled, double-blind study. J Drugs Dermatol. 2014;13(4):436-442.

12. Wu P-T, Lin C-L, Lin C-W, et al. Methylene-blue-encapsulated liposomes as photodynamic therapy nano agents for breast cancer cells. Nanomaterials. 2018;9(1):14. doi:10.3390/nano9010014

13. Klepac-Ceraj V, Patel N, Song X, et al. Photodynamic effects of methylene blue-loaded polymeric nanoparticles on dental plaque bacteria. Lasers Surg Med. 2011;43(7):600-606. doi:10.1002/ 1sm.21069

14. Lima AM, Pizzol CD, Monteiro FBF, et al. Hypericin encapsulated in solid lipid nanoparticles: phototoxicity and photodynamic efficiency. J Photochem Photobiol B. 2013;125:146-154. doi:10.1016/j. jphotobiol.2013.05.010

15. Soliman M, Salah M, Fadel M, et al. Contrasting the efficacy of pulsed dye laser and photodynamic methylene blue nanoemulgel therapy in treating acne vulgaris. Arch Dermatol Res. 2021;313 (3):173-180. doi:10.1007/s00403-020-02093-y

16. Wennersten R, Fidler J, Spitsyna A. Nanotechnology: a new technological revolution in the 21 st century. In: Misra KB, editor. Handbook of Performability Engineering. London: Springer London; 2008:943-952.

17. Piñón-Segundo E, Mendoza-Muñoz N, Quintanar-Guerrero D. Chapter 23 - nanoparticles as dental drug-delivery systems. In: Subramani K, Ahmed W, Hartsfield JK, editors. Nanobiomaterials in Clinical Dentistry. William Andrew Publishing; 2013:475-495.
18. Ferreira Soares DC, Domingues SC, Viana DB, et al. Polymerhybrid nanoparticles: current advances in biomedical applications. Biomed Pharmacother. 2020;131:110695. doi:10.1016/j. biopha.2020.110695

19. Dulińska-Litewka J, Łazarczyk A, Hałubiec P, et al. Superparamagnetic iron oxide nanoparticles - current and prospective medical applications. Materials. 2019;12(4):617. doi:10.3390/ma12040617

20. Nosrati H, Salehiabar M, Manjili HK, et al. Preparation of magnetic albumin nanoparticles via a simple and one-pot desolvation and co-precipitation method for medical and pharmaceutical applications. Int J Biol Macromol. 2018;108:909-915. doi:10.1016/ j.ijbiomac.2017.10.180

21. Repenko T, Rix A, Ludwanowski S, et al. Bio-degradable highly fluorescent conjugated polymer nanoparticles for bio-medical imaging applications. Nat Commun. 2017;8(1):470. doi:10.1038/s41467-01700545-0

22. Hathout R, Metwally A, El-Ahmady S, et al. Dual stimuli-responsive polypyrrole nanoparticles for anticancer therapy. J Drug Deliv Sci Technol. 2018;47:176-180. doi:10.1016/ j.jddst.2018.07.002

23. Rehan M, Nada AA, Khattab TA, et al. Development of multifunctional polyacrylonitrile/silver nanocomposite films: antimicrobial activity, catalytic activity, electrical conductivity, UV protection and SERS-active sensor. J Mater Res Technol. 2020;9(4):9380-9394. doi:10.1016/j.jmrt.2020.05.079

24. El-Nahrawy AM, Hammad ABA, Khattab TA, et al. Development of electrically conductive nanocomposites from cellulose nanowhiskers, polypyrrole and silver nanoparticles assisted with Nickel(III) oxide nanoparticles. React Funct Polym. 2020;149:104533. doi:10.1016/j. reactfunctpolym.2020.104533

25. Li X, Zhu X, Qiu L. Constructing aptamer anchored nanovesicles for enhanced tumor penetration and cellular uptake of water soluble chemotherapeutics. Acta Biomater. 2016;35:269-279. doi:10.1016/j. actbio.2016.02.012

26. Puiggalí-Jou A, Del Valle LJ, Alemán C. Drug delivery systems based on intrinsically conducting polymers. $J$ Control Release. 2019;309:244-264. doi:10.1016/j.jconrel.2019.07.035

27. Dan S, Feng D, Ji X, et al. Application of conducting polymers in controlled drug delivery system. Prog Chem. 2014;26:1962-1976.

28. Simya OK, Radhakrishnan P, Ashok A. Chapter 41: Engineered nanomaterials for energy applications. In: Hussain CM, editor. Handbook of Nanomaterials for Industrial Applications. Elsevier; 2018:751-767. doi:10.1016/B978-0-12-813351-4.00043-2

29. Yi N, Abidian MR. 10 - conducting polymers and their biomedical applications. In: Poole-Warren L, Martens P, Green R, editors. Biosynthetic Polymers for Medical Applications. Woodhead Publishing; 2016:243-276.

30. Guo X, Facchetti A. The journey of conducting polymers from discovery to application. Nat Mater. 2020;19(9):922-928. doi:10.1038/s41563-020-0778-5

31. Ravichandran R, Sundarrajan S, Venugopal JR, et al. Applications of conducting polymers and their issues in biomedical engineering. $J \quad R \quad$ Soc Interface. 2010;7 Suppl 5(Supp15):S559-S579. doi:10.1098/rsif.2010.0120.focus

32. Xu L, Cheng L, Wang C, et al. Conjugated polymers for photothermal therapy of cancer. Polym Chem. 2014;5(5):1573-1580. doi:10.1039/C3PY01196H

33. German N, Ramanaviciene A, Ramanavicius A. Formation and electrochemical evaluation of polyaniline and polypyrrole nanocomposites based on glucose oxidase and gold nanostructures. Polymers. 2020;12(12):3026. doi:10.3390/polym 12123026

34. German N, Ramanaviciene A, Ramanavicius A. Dispersed conducting polymer nanocomposites with glucose oxidase and gold nanoparticles for the design of enzymatic glucose biosensors. Polymers. 2021;13(13):2173. doi:10.3390/polym13132173 
35. Anderson M, Shelke NB, Manoukian OS, et al. Peripheral nerve regeneration strategies: electrically stimulating polymer based nerve growth conduits. Crit Rev Biomed Eng. 2015;43(2-3):131-159. doi:10.1615/CritRevBiomedEng.2015014015

36. Nomura S, Morimoto Y, Tsujimoto H, et al. Highly reliable, targeted photothermal cancer therapy combined with thermal dosimetry using a near-infrared absorbent. Sci Rep. 2020;10(1):9765. doi:10.1038/ s41598-020-66646-x

37. Turcheniuk K, Dumych T, Bilyy R, et al. Plasmonic photothermal cancer therapy with gold nanorods/reduced graphene oxide core/shell nanocomposites. RSC Adv. 2016;6(2):1600-1610. doi:10.1039/ C5RA24662H

38. Küünal S, Rauwel P, Rauwel E. Chapter 14 - plant extract mediated synthesis of nanoparticles. In: Barhoum A, Makhlouf ASH, editors. Emerging Applications of Nanoparticles and Architecture Nanostructures. Elsevier; 2018:411-446.

39. Vaitkuviene A, Kaseta V, Voronovic J, et al. Evaluation of cytotoxicity of polypyrrole nanoparticles synthesized by oxidative polymerization. $J$ Hazard Mater. 2013;250-251:167-174. doi:10.1016/j.jhazmat.2013.01.038

40. Vaitkuvieo A, Ratautaite V, Mikoliknaiu L, et al. Some biocompatibility aspects of conducting polymer polypyrrole evaluated with bone marrow-derived stem cells. Colloids Surf a Physicochem Eng Asp. 2014;442:152-156. doi:10.1016/j.colsurfa.2013.06.030

41. Ramanaviciene A, Kausaite A, Tautkus S, et al. Biocompatibility of polypyrrole particles: an in-vivo study in mice $\dagger$. $J$ Pharm Pharmacol. 2010;59(2):311-315. doi:10.1211/jpp.59.2.0017

42. Kong I. Chapter 7 - polymers with nano-encapsulated functional polymers. In: Thomas S, Shanks R, Chandrasekharakurup S, editors. Design and Applications of Nanostructured Polymer Blends and Nanocomposite Systems. Boston: William Andrew Publishing; 2016:125-154.

43. Fadel M, Fadeel DA, Ibrahim M, et al. One-step synthesis of polypyrrole-coated gold nanoparticles for use as a photothermally active nano-system. Int $J$ Nanomedicine. 2020;15:2605-2615. doi: $10.2147 /$ IJN.S250042

44. Rauf M, Graham J, Bukallah S, et al. Solvatochromic behavior on the absorption and fluorescence spectra of rose bengal dye in various solvents. Spectrochim Acta A. 2008;72(1):133-137. doi:10.1016/j. saa.2008.08.018

45. Li X-D, Zhai Q-Z. Evaluation of eosin Y removal from aqueous solution using nano-mesoporous material MCFs: adsorption equilibrium, kinetics, and adsorption isotherms. Int J Ind Chem. 2020;11 (1):55-67. doi:10.1007/s40090-020-00202-4

46. Fadel M, Kassab K, Youssef $T$, et al. One-step synthesis of phyto-polymer coated gold nanospheres as a delivery system to enhance resveratrol cytotoxicity. Drug Dev Ind Pharm. 2019;45 (6):937-945. doi:10.1080/03639045.2019.1579828

47. Liu W, Zhou X, Mao Z, et al. Uptake of hydrogel particles with different stiffness and its influence on HepG2 cell functions. Soft Matter. 2012;8(35):9235-9245. doi:10.1039/c2sm26001h

48. Aldalaen S, El-Gogary RI, Nasr M. Fabrication of rosuvastatin-loaded polymeric nanocapsules: a promising modality for treating hepatic cancer delineated by apoptotic and cell cycle arrest assessment. Drug Dev Ind Pharm. 2019;45(1):55-62. doi:10.1080/03639045.2018.1515221

49. Mosmann T. Rapid colorimetric assay for cellular growth and survival: application to proliferation and cytotoxicity assays. $J$ Immunol Methods. 1983;65(1-2):55-63. doi:10.1016/00221759(83)90303-4

50. Bettelheim FA, Landesberg J. Laboratory Manual for General, Organic \& Biochemistry. Saunders College Publishing; 1991.

51. Dejeu J, Taouil AE, Rougeot P, et al. Morphological and adhesive properties of polypyrrole films synthesized by sonoelectrochemical technique. Synth Met. 2010;160(23-24):2540-2545. doi:10.1016/j. synthmet.2010.10.002
52. Iram S, Zahera M, Wahid I, et al. Cisplatin bioconjugated enzymatic GNPs amplify the effect of cisplatin with acquiescence. Sci Rep. 2019;9(1):13826. doi:10.1038/s41598-019-50215-y

53. Shrikrushna S, Kulkarni J. Influence of dodecylbenzene sulfonic acid doping on structural, morphological, electrical and optical properties on polypyrrole/3C-SiC nanocomposites. J Nanomed Nanotechnol. 2015;6:1.

54. Rahaman M, Aldalbahi A, Almoiqli M, et al. Chemical and electrochemical synthesis of polypyrrole using carrageenan as a dopant: polypyrrole/multi-walled carbon nanotube nanocomposites. Polymers. 2018;10(6):632. doi:10.3390/polym10060632

55. Borlinghaus J, Albrecht F, Gruhlke $\mathrm{MCH}$, et al. Allicin: chemistry and biological properties. Molecules. 2014;19(8):12591-12618. doi:10.3390/molecules 190812591

56. Wen J, Tian Y, Mei Z, et al. Synthesis of polypyrrole nanoparticles and their applications in electrically conductive adhesives for improving conductivity. RSC Adv. 2017;7(84):53219-53225. doi:10.1039/ C7RA09725E

57. John J, Saheeda P, Sabeera K, et al. Doped polypyrrole with good solubility and film forming properties suitable for device applications. Mater Today. 2018;5(10, Part 1):21140-21146.

58. Su N. Improving electrical conductivity, thermal stability, and solubility of polyaniline-polypyrrole nanocomposite by doping with anionic spherical polyelectrolyte brushes. Nanoscale Res Lett. 2015;10 (1):301. doi:10.1186/s11671-015-0997-x

59. Ozaki J-I, Nishiyama Y. The relation between electrical conductivity and an infrared absorption parameter of carbons derived from several polymers. J Appl Phys. 1989;65(7):2744-2749. doi:10.1063/1.342763

60. Kim H-S, Seo YS, Kim K, et al. Concentration effect of reducing agents on green synthesis of gold nanoparticles: size, morphology, and growth mechanism. Nanoscale Res Lett. 2016;11(1):230. doi:10.1186/s11671-016-1393-x

61. Ibrahim A, El-Lat M, Selim M. Effect of polyethyleneimine stabilizer on size and morphology of gold nanoparticles. Asian J Appl Sci. 2016;9(4):178-184. doi:10.3923/ajaps.2016.178.184

62. Vines JB, Yoon J-H, Ryu N-E, et al. Gold nanoparticles for photothermal cancer therapy. Front Chem. 2019;7(167). doi:10.3389/ fchem.2019.00167

63. Ansari R. Polypyrrole conducting electroactive polymers: synthesis and stability studies. E- $J$ Chem. 2006;3:860413.

64. Wang F, Cai X, Su Y, et al. Reducing cytotoxicity while improving anti-cancer drug loading capacity of polypropylenimine dendrimers by surface acetylation. Acta Biomater. 2012;8(12):4304-4313. doi:10.1016/j.actbio.2012.07.031

65. Vasconcelos IB, Silva TGD, Militão GCG, et al. Cytotoxicity and slow release of the anti-cancer drug doxorubicin from ZIF-8. RSC $A d v .2012 ; 2(25): 9437-9442$. doi:10.1039/c2ra21087h

66. Zwartsen A, Hondebrink L, de Lange DW, et al. Hyperthermia exacerbates the acute effects of psychoactive substances on neuronal activity measured using microelectrode arrays (MEAs) in rat primary cortical cultures in vitro. Toxicol Appl Pharmacol. 2020;397:115015. doi:10.1016/j.taap.2020.115015

67. Seynhaeve ALB, Amin M, Haemmerich D, et al. Hyperthermia and smart drug delivery systems for solid tumor therapy. Adv Drug Deliv Rev. 2020;163-164:125-144. doi:10.1016/j.addr.2020.02.004

68. Kampinga HH. Cell biological effects of hyperthermia alone or combined with radiation or drugs: a short introduction to newcomers in the field. Int J Hyperthermia. 2006;22(3):191-196. doi:10.1080/ 02656730500532028

69. Ruiz-Pérez L, Rizzello L, Wang J, et al. Polypyrrole and polyaniline nanocomposites with high photothermal conversion efficiency. Soft Matter. 2020;16(19):4569-4573. doi:10.1039/D0SM00306A 


\section{Publish your work in this journal}

Drug Design, Development and Therapy is an international, peerreviewed open-access journal that spans the spectrum of drug design and development through to clinical applications. Clinical outcomes, patient safety, and programs for the development and effective, safe, and sustained use of medicines are a feature of the journal, which has also been accepted for indexing on PubMed Central. The manuscript management system is completely online and includes a very quick and fair peer-review system, which is all easy to use. Visit http://www. dovepress.com/testimonials.php to read real quotes from published authors. 